\title{
Primary neuroendocrine tumors of the ear, nose and throat: A report of three cases and a review of the literature
}

\author{
RACHAD MHAWEJ*, CHADI FARAH* ${ }^{*}$, AMINE HADDAD* and BASSAM TABCHY* \\ Department of Otolaryngology-Head and Neck Surgery, Hotel Dieu De France Hospital, \\ Saint Joseph University, School of Medicine, Beirut 166830, Lebanon
}

Received October 16, 2014; Accepted July 7, 2015

DOI: $10.3892 / \mathrm{ol} .2015 .3606$

\begin{abstract}
The aim of the present study was to review all cases of neuroendocrine tumors of the ear, nose and throat in a tertiary care center, as well as the data published in the literature. The study presents all the cases of neuroendocrine tumors (NETs) in the Hotel Dieu De France Hospital (Beirut, Lebanon) between January 2004 and January 2014. The data reported in the English and French literature is also reviewed with regard to the typical clinical presentation and management of these tumors. Three cases of NETs presented to the Department of Otolaryngology-Head and Neck Surgery during the study period. One case was of an atypical carcinoid (AC) tumor of the larynx, one case was of a typical carcinoid tumor in the middle ear and the third case was, to the best of our knowledge, the first reported case of an AC tumor of the nasopharynx. Overall, NETs are rare in the head and neck. The clinical presentation can mimic any other tumor in the same localization in the absence of a carcinoid syndrome. Management of these tumors remains controversial, but a complete excision of the tumor is crucial, followed by possible adjuvant treatment.
\end{abstract}

\section{Introduction}

Neuroendocrine tumors (NETs) are rare tumors arising from enterochromaffin (Kulchitsky) cells, which are part of the amine precursor uptake and decarboxylation system. The tumors are thus found in locations with a wide distribution of these cells, such as in the gastrointestinal tract, lungs and bronchi (1).

The World Health Organization (WHO) classification of NETs groups the tumors into those of epithelial origin, namely

Correspondence to: Dr Rachad Mhawej, Department of Otolaryngology-Head and Neck Surgery, Hotel Dieu De France Hospital, Saint Joseph University, School of Medicine, Alfred Naccache Street, Achrafieh, Beirut 166830, Lebanon

E-mail: rachadm@gmail.com

*Contributed equally

Key words: neuroendocrine tumors, head and neck, larynx, middle ear, nasopharynx typical (well-differentiated) carcinoid (TC) tumors, atypical (moderately-differentiated) carcinoid (AC) tumors and small cell (undifferentiated) neuroendocrine carcinoma (SmCC), and those of neural origin, namely paraganglioma (2).

The clinical and pathological features of NETs are characteristic of the organ of origin, however, these tumors can share other attributes irrespective of their anatomical site (3). Although NETs may have a similar presentation to other head and neck tumors in the same locations, their behavior and management are not clearly established and vary according to their histological type (4). Functioning tumors, particularly carcinoid NETs, can present with symptoms caused by hormone secretion; for example, patients may present with carcinoid syndrome, which is characterized by flushing, diarrhea and abdominal pain $(3,5)$. A diagnosis of carcinoid NET is suspected when features of carcinoid syndrome are present and high urinary 5-hydroxyindoleacetic acid levels are identified, with histological analyses used to confirm the diagnosis $(3,5)$. Therapeutic strategy selection depends on the site of origin of the tumor and its extent. Treatment usually consists of surgery and/or radiotherapy with or without chemotherapy (6). Rarely, NETs occur in the head and neck region, predominantly in the larynx and the middle ear, however, few cases have been described in the nasopharynx (2).

In the present study, three cases of head and neck NETs in the three different aforementioned locations are described along with their management and follow-up.

\section{Case report}

Data collection. All head and neck NET cases treated in Hotel Dieu de France Hospital (Beirut, Lebanon), between January 2004 and January 2014, were retrospectively reviewed. Data regarding clinical presentation, management, pathology results and follow-up were retrieved from hospital charts. A total of three cases were found. Written informed consent was obtained from all patients prior to surgery and prior to writing this study. A review of the English and French literature regarding NETs of the head and neck region was performed. This review was based on a search of the US National Library of Medicine (PubMed) between 1990 and 2014.

Case 1. A 64-year-old Caucasian male presented in June 2007 with hoarseness and dysphagia that had persisted for 2 years. 


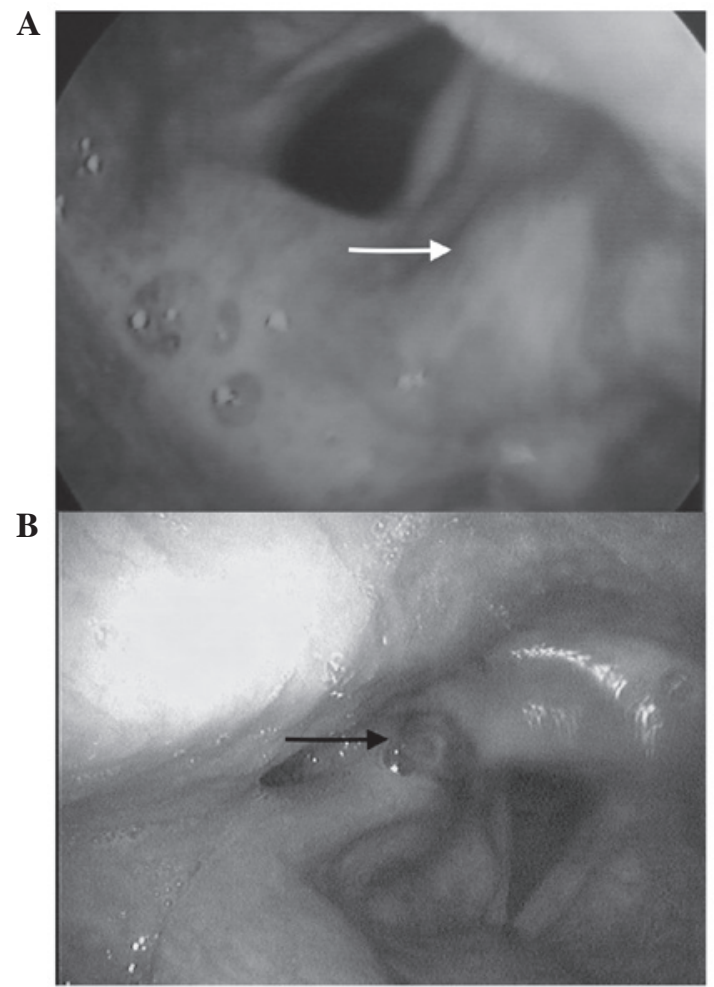

Figure 1. Flexible fibroscopy showing (A) a right arytenoid lesion (white arrow) and (B) recurrence of the tumor in the right arytenoid 5 years after the first presentation (black arrow).

The patient had smoked 2 packs of cigarettes per day for 20 years until quitting 20 years ago. The patient denied a history of alcohol consumption. The patient also experienced moderate dyspnea, particularly when in the left lateral decubitus position. A total of $20 \mathrm{~kg}$ of weight loss had been noted over the last 2 years. At 1 month prior to the current visit, the patient developed right-sided otlagia. A laryngoscopy revealed a mass in the right aryepiglottic fold (Fig. 1A). An endoscopic excisional biopsy of the lesion was performed. The tumor was composed of tubular structures and compact cords. Moderate anisonucleosis was present and the cells exhibited powder-like chromatin. Few mitotic and apoptotic cells were noted. Stroma was mildly abundant and endocrinoid in aspect. Periodic acid-Schiff coloration showed no intracytoplasmic mucosecretions. Immunohistochemistry was positive for chromogranin A, synaptophysin and cytokeratin 7 (CK7). The final diagnosis was of an AC tumor of the larynx in the right aryepiglottic fold.

Radiotherapy was suggested, but the patient refused this therapeutic option. After 3 years, recurrence of the disease was noted on the right arytenoid cartilage, which was treated by endoscopic resection. The pathology report showed recurrence of the AC tumor, with negative resection margins. The patient was then followed up regularly. At the 5-month follow-up visit, a mass was observed in the right arytenoid and aryepiglottic fold on direct laryngoscopy (Fig. 1B). Endoscopic excision of the lesion was performed using a $\mathrm{CO}_{2}$ laser. The pathology report once again showed recurrence of the $\mathrm{AC}$ tumor, with clean resection margins. The patient then underwent 28 sessions of adjuvant radiotherapy (56 Gy) for a total of 4 weeks and is currently free of disease 9 months after this treatment.

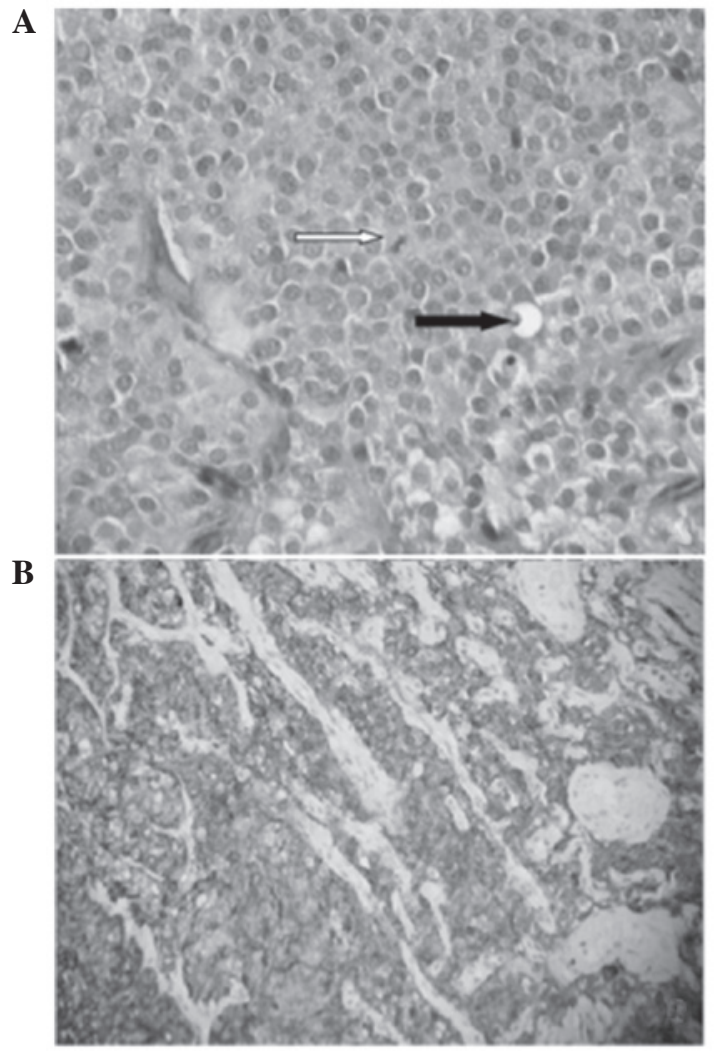

Figure 2. Pathology results: (A) High-power photomicrograph (original magnification, $\mathrm{x} 400$; hematoxylin-eosin stain) of an atypical carcinoid tumor demonstrating uniform cells with an organoid nesting pattern. One mitotic figure (white arrow) and punctuate necrosis are present in this field (black arrow). (B) Immunohistochemical staining for chromogranin A showing diffuse and intense positivity (original magnification, x200).

Case 2. A 43-year-old Caucasian female presented in July 2006 with the sensation of ear fullness and mild hearing loss that had persisted for 5 months, but with no otorrhea, vertigo or tinnitus. A physical examination showed a white retro-tympanic mass in the right ear and conductive hearing loss in the same ear, confirmed by an audiogram. A computed tomography (CT) scan showed a well-defined mass in the mesotympanum, with no erosion of the ear ossicles. The patient subsequently underwent a radical mastoidectomy with excision of the middle ear mass.

The tumor was composed of a tubular and trabecular proliferation with no patent signs of cytological malignancy. The immunohistochemistry was positive for chromogranin A and CK7. The final diagnosis was of a TC tumor of the middle ear. No clinical evidence of disease recurrence was found after 8 years.

Case 3. A 51-year-old Caucasian male presented in February 2013 with atypical vertigo that had first occurred 2 months previously. The patient had smoked 2 packs of cigarettes per day for 30 years and denied a history of alcohol consumption. No additional complaints, other than an old history of chronic nasal obstruction, were noted. Otoscopy, anterior rhinoscopy and an oral cavity examination were normal, as was the neck palpation. Brain magnetic resonance imaging was performed for the workup of the vertigo, which showed no abnormal findings in the brain parenchyma, but 
Table I. Diagnostic criteria of neuroendocrine tumors in the head and neck region.

\begin{tabular}{lccc}
\hline Criteria & TC & AC & SmCC \\
\hline Neuroendocrine differentiation & Yes & Yes & Yes \\
Mitotic count & $0-1 / 10$ HPFs & $2-10 / 10$ HPFs & $>10 / 10 \mathrm{HPFs}$ \\
Ki-67 & $<2 \%$ & $3-20 \%$ & $>20 \%$ \\
Cytoplasm amount & Moderate & Moderate & Little/scanty \\
Nucleoli & Inconspicuous & Inconspicuous & Inconspicuous \\
Nuclear polymorphism & Little & Moderate & Moderate \\
Necrosis & No & Focal punctate or mild & Marked
\end{tabular}

TC, typical carcinoid tumor; AC, atypical carcinoid tumor; SmCC, small cell neuroendocrine carcinoma; HPFs, high-power fields.

revealed the presence of a $2.2 \times 2.8-\mathrm{cm}$ mass located in the nasopharynx and in contact with the posterior region of the nasal septum. A CT scan was then performed and showed a $2.5-\mathrm{cm}$ polyp-like mass in the nasopharynx. The mass was of tissular density and contained small calcifications, with no visible adenopathies on CT scan. A subsequent endoscopic resection of the mass was performed.

Histopathologically, the tumor was composed of an endocrinoid proliferation containing sheets and nests separated by a thin vascularized stroma. The tumor cells exhibited round nuclei containing salt and pepper-like chromatin. Cytoplasm was abundant and amphophilic. Mild nuclear pleomorphism was noted and punctuate areas of necrosis were present. Mitotic activity was low with a rate of $1 / 10$ high-power fields (Fig. 2A). Immunohistochemistry was diffusely positive for chromogranin A (Fig. 2B), synaptophysin and pancytokeratin. There was no S100 staining of the tumor cells, nor of the sustentacular cells. Calcitonin, thyroid transcription factor-1 and caudal-type homeobox- 2 staining was also negative. The proliferation index was $3 \%$. The final diagnosis was of an AC tumor of the nasopharynx.

Positron emission tomography-CT scanning with gallium-68-DOTA-NOC was performed 1 month post-operatively to search for other localizations and revealed positive uptake in the nasopharynx only. The urinary 5-hydroxyindoleacetic acid level was $9.1 \mathrm{mg} / 24 \mathrm{~h}$ (normal range, 2-6 mg/24 h). At 3 months post-surgery, the patient was administered 28 sessions of adjuvant radiotherapy (56 Gy) for a total of 4 weeks, and is currently free of disease at 14 months of follow-up.

\section{Discussion}

Neuroendocrine neoplasms are rare tumors that are mainly found in the GI tract, pancreas and lungs (5).

Numerous proposals have been put forward with regard to the classification and nomenclature of NETs, and a number of these differ in their use of specific terminology and the criteria for grading and staging (3). In order to predict the patient outcome and therapy, the use of a single system of nomenclature, grading and staging is required for NETs of all anatomical sites, as there are a number of similarities among NETs throughout the body. However, certain systems that have arisen independently have become firmly established and are recognized by organizations charged with standardizing terminology, such as the World Health Organization (WHO). No data favors one system over another (3). The basic data that underlie the systems are similar, even if the criteria differ.

NETs are rarely found in the head and neck, particularly the larynx, the middle ear and the nasopharynx. The WHO classification of laryngeal and middle ear NETs is generally consistent with that for pulmonary neuroendocrine carcinoma. The tumors can be subdivided into TC, AC, SmCC, combined SmCC with non-small cell carcinoma, and those with neurological origins, termed paragangliomas, according to the 2005 WHO classification of head and neck tumors $(2,6-8)$. With regard to large cell neuroendocrine carcinomas, while the WHO classification does not distinguish them from AC, they have been considered as a separate subtype by one previous study (6). However, NETs of the nasopharynx are not included in any of the WHO classifications of nasopharyngeal tumors.

Histological grading depends on the number of mitoses, the presence of necrosis and the Ki-67 index (Table I) $(2,3,6,7)$.

In the head and neck region, NETs are mostly found in the larynx. To date, $\sim 650$ patients with neuroendocrine neoplasms of the larynx have been reported in the literature (9). The most frequent type is the AC tumor, followed by $\mathrm{SmCC}$, paraganglioma and finally, the TC tumor (10).

NETs rarely occur in the middle ear, but this location is considered second in frequency after the larynx when it comes to the head and neck region. Approximately 50 cases $(11,12)$ of carcinoid tumor of the middle ear have been reported in the English literature (11). However, neuroendocrine cells have not been demonstrated specifically within the middle ear, and the precise origin of carcinoid tumor of the middle ear has not been clarified (11).

NETs can also rarely occur in the nasal cavity or the nasopharynx. In the nasopharynx, cases reported in the literature were either SmCC or TC, with no cases of AC previously reported. To the best of our knowledge, case 3 of the present study is the first described case of a nasopharyngeal AC tumor.

The mainstay therapy for all NETs of the larynx, with the exception of SmCC, is surgical excision. Depending on the site and extent of the primary tumor, a partial or total laryngectomy may be performed (13). In a recent systematic review and meta-analysis of all laryngeal neuroendocrine carcinomas, van der Laan et al reviewed treatment schemes according to tumor subtypes and resulting survival rates. 
The study found that the more benign end of the spectrum is represented by TC tumors, and that local excision alone is curative. Radiotherapy does not induce a good response in patients with AC tumors, which are therefore best managed by radical surgical excision in combination with elective bilateral neck dissection due to the high propensity for regional metastasis. The most benefit for cases of SmCC or large cell neuroendocrine carcinoma appears to be gained from a combination of radiotherapy and chemotherapy, although patient survival remains poor (14).

Laryngeal paragangliomas are almost always benign and should be treated accordingly. Partial laryngectomy is preferable to radiation as a cure is usually achieved without loss of laryngeal function (15). Laser surgery is not widely used due to the vascular nature of these tumors (13).

Carcinoid tumors of the middle ear are primarily treated by surgical excision. There is no known established surgical approach, perhaps owing to the limited incidence of the condition, but complete removal by tympano-mastoidectomy or radical mastoidectomy is the most commonly used technique (16). In the literature, middle ear carcinoid tumors were successfully removed by tympanotmy alone in certain studies, while in other cases, a subtotal petrosectomy was performed (17). No sufficient data exist regarding the role of chemotherapy or radiation therapy in middle ear carcinoid tumors (17). Radiation therapy was used as adjuvant therapy in certain studies, but the number of cases was insufficient to draw a conclusion concerning better local control and recurrence rates (18).

In view of the small number of cases, no clear treatment policy has been established for neuroendocrine carcinomas of the nasal cavity, paranasal sinuses and nasopharynx. In almost all the cases reported in the English literature, the treatment consisted of surgery (7,19-25). In certain studies, conventional radiotherapy was used alone or in combination with either surgery or chemotherapy, but the efficacy of such regimens is yet to be proven (22). In a review by Furuta et al, it was proposed that radiation therapy and chemotherapy should be performed as post-operative adjuvant therapy when a complete resection is difficult and when surgery is ineffective (24).

NETs are rarely diagnosed in the head and neck region. The tumors are found mainly in the larynx, less frequently in the middle ear and scarcely in the nasal cavity or nasopharynx. While a well-defined classification exists for NETs of the larynx and the middle ear, these tumors are not mentioned in the most recent WHO classification of nasopharyngeal tumors. Clinicians should be aware of this tumor in the nasopharynx and should consider it in the differential diagnosis of tumors located there. Following the recent meta-analysis by van der Laan et al (14), treatment schemes for laryngeal NETs have been well established considering the low level of evidence on this subject. However further studies are required to establish treatment protocols for the middle ear and nasopharyngeal locations.

\section{References}

1. Bapat U, Mackinnon N and Spencer MG: Carcinoid tumours of the larynx. Eur Arch Otorhinolaryngol 262: 194-197, 2005.
2. Barnes L, Eveson $J$, Reichart $P$ and Sidransky DE: Neuroendocrine tumours. In: World Health Organization Classification of Tumours: Pathology and Genetics of Head and Neck Tumours. IARC Press, Lyon, pp135-139, 2005.

3. Klimstra DS, Modlin IR, Coppola D, Lloyd RV and Suster S: The pathologic classification of neuroendocrine tumors: A review of nomenclature, grading and staging systems. Pancreas 39: 707-712, 2010.

4. Meacham R, Matrka L, Ozer E, Ozer HG, Wakely P and Shah M: Neuroendocrine carcinoma of the head and neck: A 20-year case series. Ear Nose Throat J 91: E20-E24, 2012.

5. Kunz PL: Carcinoid and neuroendocrine Tumors: Building on success. J Clin Oncol 33: 1855-1863, 2015.

6. Kao H-L, Chang W-C, Li W-Y, Chia-Heng Li A and Fen-Yau $\mathrm{Li} \mathrm{A}$ : Head and neck large cell neuroendocrine carcinoma should be separated from atypical carcinoid on the basis of different clinical features, overall survival and pathogenesis. Am J Surg Pathol 36: 185-192, 2012.

7. Weinreb I and Perez-Ordoñez B: Non-small cell neuroendocrine carcinoma of the sinonasal tract and nasopharynx. Report of 2 cases and review of the literature. Head Neck Pathol 1: 21-26, 2007.

8. Lewis JS Jr, Ferlito A, Gnepp DR, Rinaldo A, Devaney KO, Silver CE and Travis WD; International Head and Neck Scientific Group: Terminology and classification of neuroendocrine neoplasms of the larynx. Laryngoscope 121: 1187-1193, 2011

9. Ferlito A and Rinaldo A: The spectrum of endocrinocarcinomas of the larynx. Oral Oncol 41: 878-883, 2005.

10. Ferlito A, Devaney KO and Rinaldo A: Neuroendocrine neoplasms of the larynx: Advances in identification, understanding and management. Oral Oncol 42: 770-788, 2006.

11. Tabuchi K, Aoyagi Y, Uemaetomari I, Tobita T, Wada T, Inadome $\mathrm{Y}$, Noguchi $\mathrm{M}$ and Hara A: Carcinoid tumours of the middle ear. J Otolaryngol Neck Surg 38: E91-E94, 2009.

12. Bittencourt AG, Tsuji RK, Cabral F Junior, Pereira LV, Fonseca AC, Alves V and Bento RF: Middle ear adenoma with neuroendocrine differentiation: Relate of two cases and literature review. Int Arch Otorhinolaryngol 17: 340-343, 2013.

13. Ferlito A, Silver CE, Bradford CR and Rinaldo A: Neuroendocrine neoplasms of the larynx: An overview. Head Neck 31: 1634-1646, 2009.

14. van der Laan TP, Plaat BE, van der Laan BF and Halmos GB: Clinical recommendations on the treatment of neuroendocrine carcinoma of the larynx: A meta analysis of 436 reported cases. Head Neck 37: 707-715, 2014.

15. Myssiorek D, Rinaldo A, Barnes L and Ferlito A: Laryngeal paraganglioma: An updated critical review. Acta Otolaryngol 124: 995-999, 2004.

16. Baig S, Patil N and Considine N: Carcinoid tumour of the middle ear. J Coll Physicians Surg Pak 22: 604-606, 2012.

17. Ramsey MJ, Nadol JB Jr, Pilch BZ and McKenna MJ: Carcinoid tumor of the middle ear: Clinical features, recurrences and metastases. Laryngoscope 115: 1660-1666, 2005.

18. Krouse JH, Nadol JB and Goodman ML: Carcinoid tumors of the middle ear. Ann Otol Rhinol Laryngol 99: 547-552, 1990.

19. Galm T and Turner N: Primary carcinoid tumour of nasal septum. J Laryngol Otol 123: 789-792, 2009.

20. Kanoh N, Nishimura Y, Nakamura M, Mori M and Uematsu K: Primary nasopharyngeal paraganglioma: A case report. Auris Nasus Larynx 18: 307-314, 1991.

21. Li AL, Wehrli B and Rotenberg BW: Carcinoid tumour arising simultaneous to an inverted papilloma in the nasal cavity. J Otolaryngol Head Neck Surg 39: E78-E82, 2010.

22. Vandist V, Deridder F, Waelput W, Parizel PM, Van de Heyning P and Van Laer C: A neuroendocrine tumour of the sphenoid sinus and nasopharynx: A case report. B-ENT 6: 147-151, 2010.

23. Chu MW, Karakla DW, Silverberg M and Han JK: Primary carcinoid tumor of the frontal sinus: A case report. Ear Nose Throat J 89: E13-E16, 2010.

24. Furuta A, Kudo M, Kanai K, Ohki S and Suzaki H: Typical carcinoid tumor arising in the nose and paranasal sinuses-case report. Auris Nasus Larynx 37: 381-385, 2010.

25. Lee DH, Cho HH and Cho YB: Typical carcinoid tumor of the nasal cavity. Auris Nasus Larynx 34: 537-539, 2007. 